\title{
Partial cross sections of doubly excited helium below the ionization threshold $I_{7}$
}

\author{
Y. H. Jiang, ${ }^{1,2}$ R. Püttner, ${ }^{1}$ R. Hentges,${ }^{3}$ J. Viefhaus,${ }^{3}$ M. Poiguine, ${ }^{1}$ U. Becker, ${ }^{3}$ J. M. Rost,${ }^{2}$ and G. Kaindl ${ }^{1}$ \\ ${ }^{1}$ Institut für Experimentalphysik, Freie Universität Berlin, Arnimallee 14, D-14195 Berlin-Dahlem, Germany \\ ${ }^{2}$ Max-Planck-Institut für Physik Komplexer Systeme, Nöthnitzer Straße 38, D-01187 Dresden, Germany \\ ${ }^{3}$ Fritz-Haber-Institut Berlin, Faradayweg 4-6, D-14195 Berlin-Dahlem, Germany
}

(Received 5 December 2003; published 6 April 2004)

\begin{abstract}
Partial photoionization cross sections (PCSs), $\sigma_{n}$, leading to final ionic states of helium, $\mathrm{He}^{+}(n)$, were measured at BESSY II in the region of doubly excited helium up to the ionization threshold $I_{7}$ of $\mathrm{He}^{+}$. The experiments were performed with a time-of-flight (TOF) electron spectrometer and high photon resolution, $\Delta E \cong 6 \mathrm{meV}$. The results of these measurements are a most critical assessment of the decay dynamics of double-excitation resonances and agree well with those of recent eigenchannel $R$-matrix calculations. They also confirm the propensity rules set up for the autoionization of doubly excitated states. The mirroring behavior in the PCSs predicted recently by Liu and Starace is only partially observed. By discussing the formulas given by these authors in a more general context, the specific behavior of the PCSs of helium with respect to mirroring can be understood. The mirroring compensation properties between the "fractional partial cross sections" $\gamma_{P}$ $=\sigma_{P} / \sigma_{T}$ and $\gamma_{Q}=\sigma_{Q} / \sigma_{T}$, with $\sigma_{T}=\sigma_{P}+\sigma_{Q}$, are introduced and discussed.
\end{abstract}

DOI: 10.1103/PhysRevA.69.042706

PACS number(s): $32.80 . \mathrm{Fb}, 32.80 . \mathrm{Dz}$

\section{INTRODUCTION}

Double-excitation states in helium have been considered to be prototypical for two-electron systems with strong electron correlation since their observation by Madden and Codling in the 1960s, [1]. With the advance of third-generation synchrotron radiation facilities and the availability of high resolving power and brightness, high resolution measurements [2,3] along with advanced theoretical approaches [4-6] emerged for the region of doubly excited helium. In the energy region above the fourth ionization threshold of $\mathrm{He}^{+}, I_{4}$, the most interesting features are caused by pertubers; these are low-lying resonances of Rydberg series belonging to higher ionization thresholds, which interfere with Rydberg series that converge towards a lower ionization threshold. Predicted by quantum defect theory [7], these pertubers will influence the energy positions of Rydberg resonances by an increase of the quantum defect by one within the region of the interference; the linewidths of the corresponding Fano resonances are also modulated. In the region close to the double ionization threshold, the interferences due to the overlap of several pertubers with different Rydberg series render the observed spectra highly complicated. In this case, the approximate quantum numbers begin to lose their meaning, and the regularities in the two-electron resonance spectrum start to dissolve. The statistical properties of the spacings between neighboring energy levels display a transition towards quantum chaos [3]. For this reason, the last electron volt below the double ionization threshold has drawn considerable attention in both experimental and theoretical studies. But so far, these studies have mainly been restricted to total cross sections (TCSs) [2-6].

First measurements of partial cross sections (PCSs) were performed by Lindle et al. [8]; these measurements, however, were limited to excitations approaching the ionization limit $I_{3}$ owing to the low photon intensities achieved at the first-generation light sources of that time. Menzel et al. reported on measurements of partial cross sections up to the ionization threshold $I_{5}$ employing two spherical-sector-plate electrostatic analyzers $[9,10]$ and discussed the observed general patterns in $\sigma_{n}$. Here, $\sigma_{n}$ describes the PCS for the channel leading to $\mathrm{He}^{+}$, with the single electron in a state with principal quantum number $n$. Very recently, Schneider et al. [11] and van der Hart et al. [12] confirmed in their calculations these regularities in $\sigma_{n}$ up to the ionization threshold $I_{9}$. The similarities in the PCSs for the energy region with a small number of pertubers, also called "general patterns," can be well understood with the so-called propensity rules based on the molecular adiabatic approximation [11]. As the photon energy increases, the structures in the PCS get more and more complicated due to interferences caused by an increasing number of pertubers, and the general patterns begin to dissolve. A confirmation of these theoretical predictions requires high-resolution photoemission measurements at energies beyond the ionization threshold $I_{5}$.

The mirroring behavior of PCSs for high-lying doubleexcitation resonances has become an interesting topic in recent years since Liu and Starace developed an analytical expression [13]. In general, the TCS consists of a number PCSs which are defined by the quantum numbers of the outgoing electron and the remaining ion. After dividing the PCSs in any two groups, labeled $P$ and $Q$, the analytical expression predicts that the sum of one group of PCSs, $\sigma_{P}$, exhibitsclose to the resonances-a mirroring behavior to the sum of the other group of PCSs, $\sigma_{Q}$, if $\rho^{2} \rightarrow 0$; here, the energy axis is taken as the mirror plane [see, e.g., Fig. 6(c)] and $\rho^{2}$ is the fractional part of the TCS that interacts with the resonance [14]. As a result of this mirroring behavior, a resonance that is almost invisible in the TCS $\left(\rho^{2} \rightarrow 0\right)$ can lead to strong variations in $\sigma_{P}$ and $\sigma_{Q}$. This has been shown by CantonRogan and co-workers for two resonances of doubly excited Ar that are extremely weak in the TCS; in the PCSs, however, the resonances are clearly visible [15]. Although $\rho^{2}$ $\rightarrow 0$ is required for a mirroring behavior to occur, it has been observed in the theoretical photoionization cross section of 
$\mathrm{Li}^{-}$even for $\rho^{2}$ as high as 0.3 [13]. In contrast to this, for helium in the double excitation region two sets of PCSs do not always mirror each other even for $\rho^{2} \cong 0.01$.

In the present work, we present experimental results for PCSs in the region of doubly excited helium up to the $N$ $=7$ ionization threshold of $\mathrm{He}^{+}$. These PCSs were derived from photoemission spectra taken by a time-of-flight (TOF) electron spectrometer. In addition, the PCSs were also calculated by state-of-the-art $R$-matrix calculations and the analytical expression given previously by Liu and Starace for the mirroring behavior of PCSs was extended to a more general case. This allows an explanation of the behavior observed in the vicinity of double-excitation resonances of helium. In addition, the mirroring compensation properties in the fractional PCSs are discussed. The incentive to the present work is the insight that an analysis of the propensity rules and the mirroring behavior of PCSs of autoionizing double-excitation states of helium is expected to provide a deep understanding of the decay dynamics of two-electron atoms.

\section{EXPERIMENTAL SETUP AND PROCEDURE}

State-of-the-art high-resolution monochromators in combination with TOF photoelectron spectrometers allow one to explore the autoionization of resonances in the region close to the double-ionization threshold of helium. Here, we present photoemission measurements up to the ionization threshold $I_{7}$ and extract the various PCSs from the recorded spectra in this energy region. The experiments were performed at the undulator beamline U125/2-SGM (BUSbeamline) [16] of the Berliner Elektronenspeicherring für Synchrotronstrahlung (BESSY) using a photon energy resolution of $\Omega \cong 6 \mathrm{meV}$ [full width at half maximum (FWHM)]. The TOF spectrometer [17] was mounted at the magic angle, i.e., in the dipole plane at an angle of $\theta=55^{\circ}$ relative to the polarization direction of the incoming light. In this geometry, the angular distribution can be assumed to cause no effects. A needle (10 cm long, less than $500 \mu \mathrm{m}$ inner diameter) directs an effusive jet of gas to the interaction region; the background pressure in the chamber was $\cong 10^{-4}$ mbar. From the count rate and calculated cross sections we estimated the pressure in the interaction region to be of the order of $10^{-2}$ mbar. TOF photoelectron spectra were taken for each photon energy and converted into photoemission spectra (PES) by a time-to-energy conversion procedure.

As an example, an experimental photoemission spectrum, taken with $h \nu=76.2 \mathrm{eV}$, is presented in Fig. 1 in the form of a three-dimensional plot. From the PES the integral intensity of the satellite peaks, i.e., $n>1$, can be extracted and plotted as a function of photon energy, resulting in the PCS $\sigma_{n}$. As shown in Fig. 1 for the example of $\sigma_{3}$, one PCS is normally derived from approximately $100 \mathrm{PES}$; this procedure requires data acquisition for several tens of hours. For more details see Ref. [18]. In order to eliminate the influences caused by the decrease of the ring current and fluctuations of the gas pressure in the interaction region during data taking, the spectra were normalized to the intensity of the $n=1$ line, i.e., the partial cross section $\sigma_{1}$ is assumed to be without

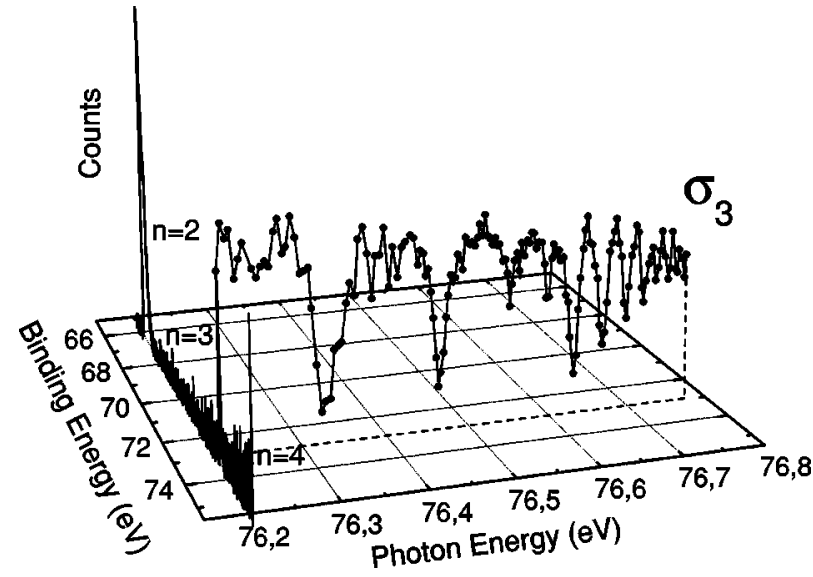

FIG. 1. The bar diagram along the binding energy axis represents an experimental photoemission spectrum measured with $h \nu$ $=76.2 \mathrm{eV}$, with the satellites $n=2$ to 4 representing the ionic final states of $\mathrm{He}^{+}(n)$. The solid curve through the data points represents the PCS $\sigma_{3}$ that can be derived from a large number of such photoemission spectra (not shown here) recorded at different photon energies. For details see text.

structure. Based on theoretical results, which show some weak resonances in $\sigma_{1}$, we can estimate that the error produced by this procedure is $\leqslant 10 \%$ for measurements below the ionization threshold $I_{5}, \leqslant 6 \%$ below $I_{6}$, and $\leqslant 7 \%$ below $I_{7}$.

\section{THEORETICAL METHOD}

The eigenchannel $R$-matrix method [19], combined with a close-coupling scheme [20], is employed for calculating the partial cross sections. The configuration space is partitioned into the reaction region (defined by $r_{i}<r_{0}$, where $r_{i}$ are the electron distances from the nucleus) and the external region $\left(r_{i}>r_{0}\right)$. In the reaction region, the full two-electron problem is solved numerically by bound-state configurationinteraction techniques to obtain the so-called eigenchannel wave functions. These wave functions are linearly combined into helium wave functions of experimentally observed channels. In the external region, a multichannel wave function with one bound and one continuum wave function is again linearly combined into a helium eigenfunction according to the incoming-wave boundary condition [20]. By matching the linear combinations of the multichannel basis functions for the two regions, one can determine an accurate final-state wave function $\Psi_{f}^{(-)}$, which describes the experimentally observed channel $f$. The partial cross section for absorbing a photon of energy $h \nu$ from the initial state $\Psi_{0}$ (here the ground state of helium) reads [21]

$$
\sigma_{f}=\frac{8 \pi^{3} \nu}{c}\left|\left\langle\Psi_{f}^{(-)}|D| \Psi_{0}\right\rangle\right|^{2}
$$

with the dipole operator $D$, and the speed of light $c$. For the results presented here, a radius of $r_{0}=200$ a.u. was used, and a total of 1080 closed-type (i.e., zero at the radius $r_{0}$ ) and 20 open-type (i.e., nonzero at the radius $r_{0}$ ) one-electron 
wave functions with orbital angular momenta up to nine were included. 9610 closed-type two-electron configurations were included in the calculation for the final-state wave function. For each channel, in which one electron can escape from the reaction region, two open-type orbitals for the outer electron were included in addition to the closed-type basis set [11].

\section{EXPERIMENTAL RESULTS AND DISCUSSION}

\section{A. Classification of the ${ }^{1} P^{o}$ resonances in doubly excited helium}

The double-excitation ${ }^{1} P^{o}$ resonances of helium can be identified in a simplified classification scheme $N, K_{n^{\prime}}$ [22,23], with $N$ and $n^{\prime}$, respectively, standing for the ionization threshold of a given channel and the running index of the considered Rydberg series; $K$ represents the angularcorrelation quantum number. In an independent particle picture, $N\left(n^{\prime}\right)$ can be understood as the quantum number of the inner (outer) electron. The Stark quantum numbers $\left[N_{1} N_{2} m\right]$ (also called parabolic quantum numbers) provide an alternative, but equivalent classification scheme for two-electron states. It is easy to understand this latter classification scheme in the limit of almost singly ionized two-electron states. There, the outer electron is so far away from the nucleus that it merely creates an electric field for the inner electron whose eigenstate becomes a single-electron Stark state. A change in individual Stark quantum numbers reflects directly the autoionization process of a two-electron system and describes the resonance properties of the different decay channels $n$. Therefore, Stark quantum numbers are more convenient when discussing the general patterns in $\sigma_{n}$. The two classification schemes $\left[N_{1} N_{2} m\right]_{n^{\prime}}$ and $N, K_{n^{\prime}}$ are related by $K=N_{2}-N_{1}$ and $N=N_{1}+N_{2}+m+1$, with $m$ being the projection of the angular momentum on the axis defined by the two electrons. A more detailed discussion of these quantum numbers is given in Ref. [5]. In the present publication we use both classification schemes.

\section{B. Experimental results and data analysis}

The resonance profiles in the TCS can be described by the Fano formula [24]

$$
\sigma_{T}=\sigma_{T}^{0}\left(\rho^{2} \frac{(q+\epsilon)^{2}}{1+\epsilon^{2}}+1-\rho^{2}\right) \text { with } \epsilon=2 \frac{E-E_{r}}{\Gamma} .
$$

Here, $E_{r}, \Gamma$, and $q$ stand for the resonance energy, the natural width, and the Fano $q$ parameter, respectively; $\sigma_{T}^{0}$ is the TCS in the absence of resonances. Note that the Fano formula, which had been developed for describing the line shapes of resonances in the TCS, has the same mathematical structure as the exact formula given by Starace for describing the resonances in the PCS [25]. As a consequence, it is possible to describe the PCSs by the Fano formula, but in this case $q$ represents only an effective parameter without deeper meaning. In analyzing our data, we fitted the various PCSs for a given energy region by the Fano formula in a parallel leastsquares fit procedure using a single Gaussian to simulate the experimental resolution function. In this way, the $\sigma_{n}$ leading

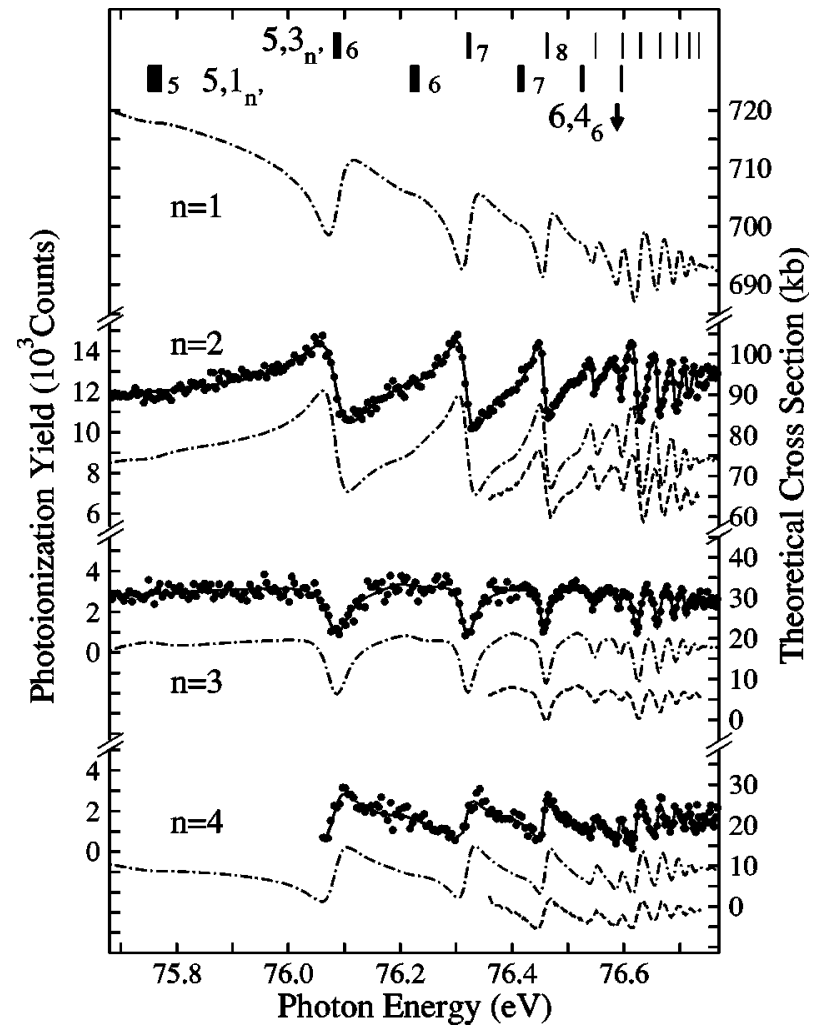

FIG. 2. Experimental partial cross sections $\sigma_{n}^{5}$ leading to the final states $\operatorname{He}^{+}(n)$, with $n=2,3$, and 4, along with the results of the eigenchannel $R$-matrix calculations. The data were taken in the photon-energy region between $75.68 \mathrm{eV}$ and $76.77 \mathrm{eV}$. The two vertical-bar diagrams in the upper part of the figure give the assignments of the double-excitation resonances by specifying $n^{\prime}$; with the widths of bars being proportional to the linewidths of the corresponding resonances [5]. The vertical arrow in the upper right corner marks the calculated energy of the perturber $6,4_{6}$. The filled data points are the present experimental results, with the solid curves through the data points representing the fit results. The dashed curves represent the data by Menzel et al. [9] and the dashed-dotted curves the results of the $R$-matrix calculations convoluted with a Gaussian of $6 \mathrm{meV}$ width (FWHM) to simulate finite experimental resolution.

to different final states of $\mathrm{He}^{+}(n)$ were fitted to Eq. (2) with the same experimental resolution $\Omega$, energy position $E_{r}$, and linewidth $\Gamma$, but different Fano $q$ parameters. The results of this fit procedure are given as solid curves through the data points in Figs. 2-5. The data presented here were calibrated at the energy of resonance 5,36 $(76.083 \mathrm{meV})$ as given by Domke et al. [2].

Note that for convenience we employ in our discussion the notation $\sigma_{n}^{N}$ to label the different PCSs. As defined above, the lower index $n(n=1,2,3, \ldots, N-1)$ refers to the principal quantum number of the single electron in the ionized final state, $\mathrm{He}^{+}(n) . N$ is the quantum number of the inner electron in the doubly excited states, i.e., the corresponding data are measured below the ionization threshold $I_{N}$.

Figure 2 displays the results of this work for the PCSs $\sigma_{n}^{5}$ (with $n=2,3$, and 4) along with the results of the described least-squares-fit analysis (filled data points and solid curves), 


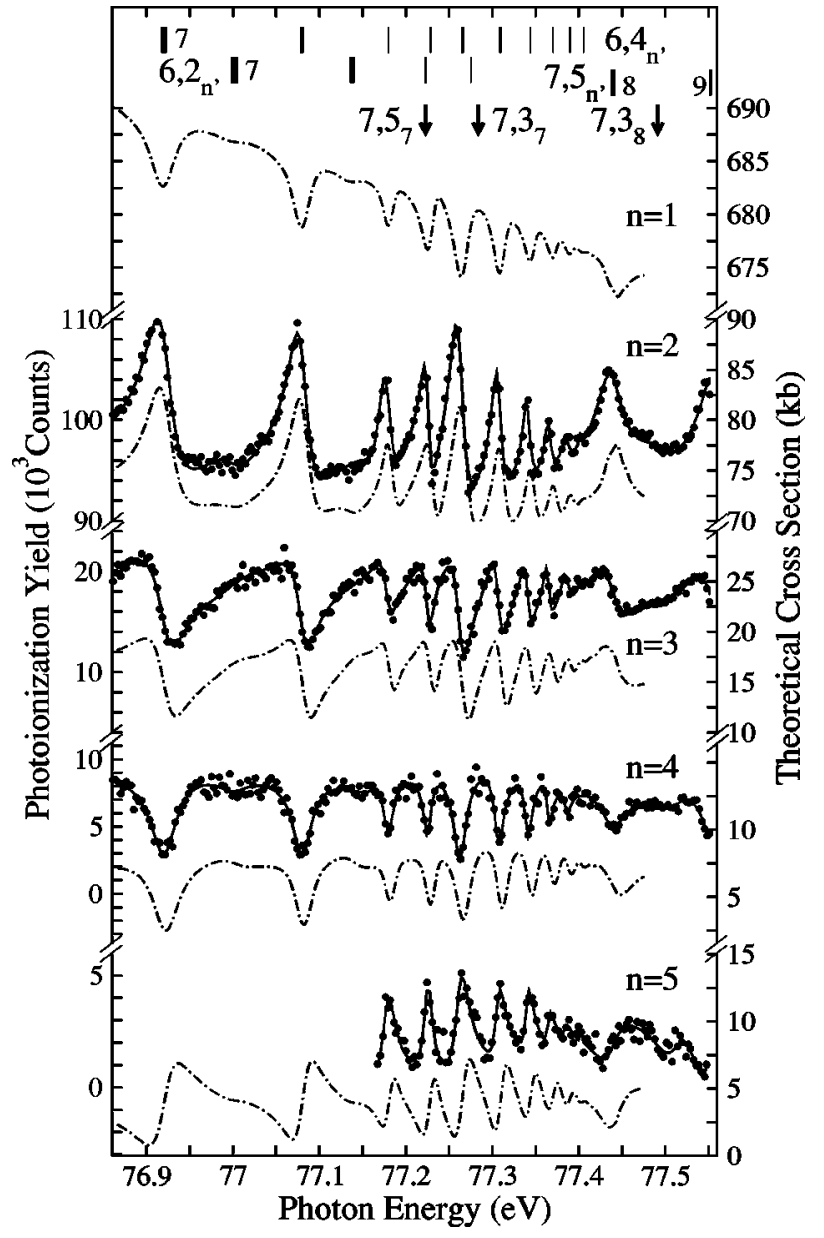

FIG. 3. Experimental partial cross sections $\sigma_{n}^{6}$ leading to the final states $\mathrm{He}^{+}(n)$, with $n=2,3,4$, and 5, respectively, in the energy region from $76.85 \mathrm{eV}$ to $77.56 \mathrm{eV}$, along with the results of the eigenchannel $R$-matrix calculations; for details, see the caption of Fig. 2.

the results of the eigenchannel $R$-matrix calculations and previous results of Ref. [9]. The error bars in the cross sections were estimated to be $\pm 2 \%$ for $\sigma_{2}^{5}, \pm 10 \%$ for $\sigma_{3}^{5}$, and $\pm 15 \%$ for $\sigma_{4}^{5}$. This behavior can be readily understood with the following facts: (i) the lower decay channels have larger cross sections; (ii) the corresponding photoelectrons have higher kinetic energies, leading hence to a higher transmission through the TOF spectrometer. Both facts give rise to higher count rates and therefore to smaller error bars. The principal series $5,3_{n^{\prime}}$ is observed up to resonance $n^{\prime}=15$. The present relative measurements agree well with those of Menzel et al. where line shapes and energy positions of the resonances in all three decay channels are concerned. The previously unobserved resonances $5,3_{6}$ and $5,3_{7}$ in $\sigma_{3}^{5}$ and $\sigma_{4}^{5}$, respectively, are related to photoelectrons with relatively low kinetic energies, demonstrating the enhanced capability of the TOF analyzers used here in detecting photoelectrons with kinetic energies as low as $350 \mathrm{meV}$ at sufficiently high count rates. In the $n=2$ channel, the resonances $n^{\prime}=6,7$, and 8 of the secondary series $5,1_{n^{\prime}}$ could be detected in the fit analysis with the present monochromator resolution of $\cong 6 \mathrm{meV}$ (FWHM).

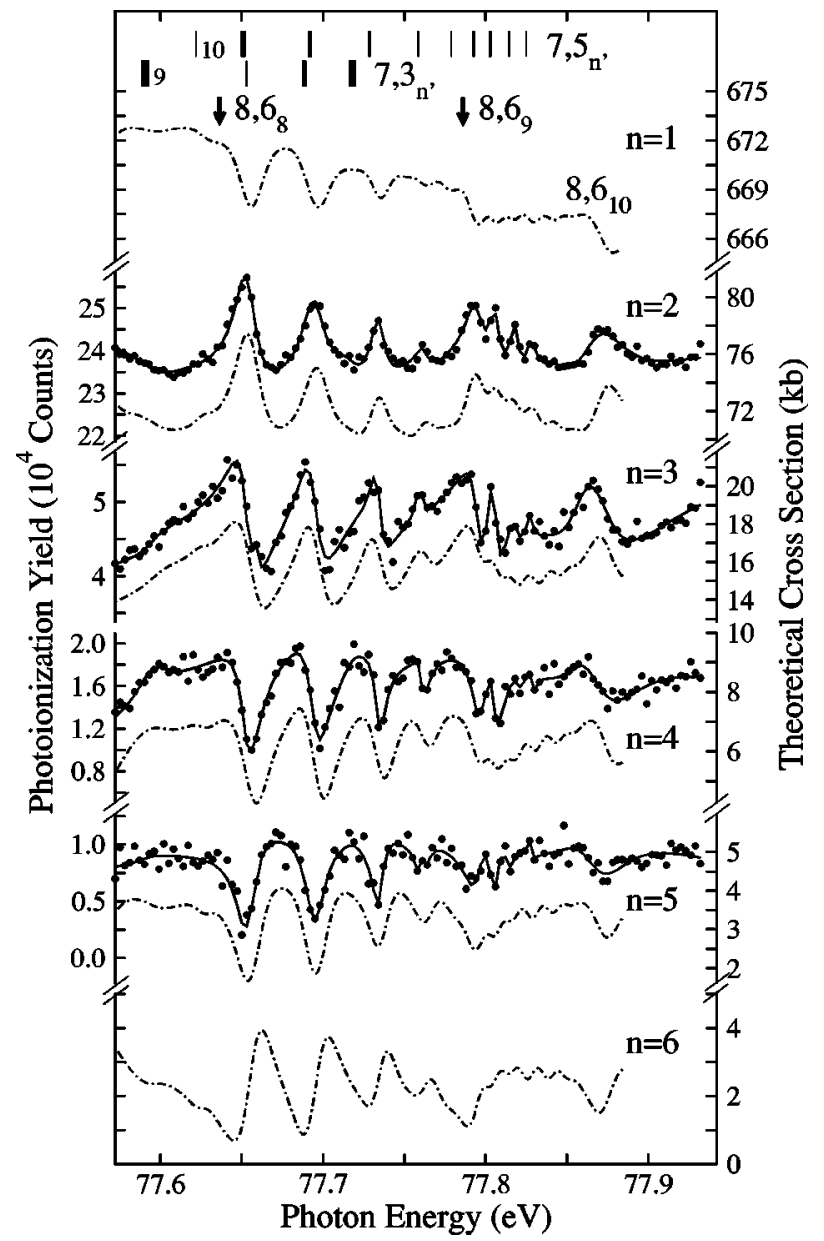

FIG. 4. Experimental partial cross sections $\sigma_{n}^{7}$ leading to the final states $\mathrm{He}^{+}(n)$, with $n=2,3,4$, and 5, respectively, in the energy region from $77.56 \mathrm{eV}$ to $77.92 \mathrm{eV}$, along with the results of the eigenchannel $R$-matrix calculations. Note that the plotted theoretical results were shifted by $12 \mathrm{meV}$ to lower energies; for details, see the caption of Fig. 2.

The PCSs below the ionization threshold $I_{6}$, with the perturbers $7,5_{8}$ and $7,5_{9}$, and below the ionization threshold $I_{7}$, with the perturber $8,6_{10}$, are shown in Figs. 3 and 4 , respectively. The principal series $6,4_{n^{\prime}}$ and $7,5_{n^{\prime}}$ are observed up to the resonances $n^{\prime}=16$ and 19 , respectively. Note that the resonances $7,5_{10}$ and $7,5_{15}$ are almost completely suppressed due to interferences with the perturbers $8,6_{8}$ and $8,6_{9}$, respectively. These findings agree well with the results of earlier studies of the total absorption cross sections [2]. The error bars in the PCS curves were estimated to be $\pm 1 \%\left(\sigma_{2}^{6}\right), \quad \pm 1 \%\left(\sigma_{2}^{7}\right), \quad \pm 3 \%\left(\sigma_{3}^{6}\right), \quad \pm 2 \%\left(\sigma_{3}^{7}\right), \quad \pm 6 \%\left(\sigma_{4}^{6}\right)$, $\pm 5 \%\left(\sigma_{4}^{7}\right), \pm 11 \%\left(\sigma_{5}^{6}\right)$, and $\pm 10 \%\left(\sigma_{5}^{7}\right)$. Again, the resonances $6,2_{7}, 6,2_{8}$, and $7,3_{8}$ of the secondary series are observed in the fit analysis of $\sigma_{2}^{6}$ and $\sigma_{4}^{7}$, respectively. Due to small partial cross sections and a relatively low transmission rate of the TOF spectrometer for slow electrons, the PCS $\sigma_{5}^{6}$ in the energy region below $77.155 \mathrm{eV}$ (Fig. 3) and the PCS $\sigma_{6}^{7}$ (Fig. 4) could not be obtained from the present measurements.

The dashed-dotted curves in Figs. 2-4 represent the results of $R$-matrix calculations convoluted with a Gaussian of $6 \mathrm{meV}$ width (FWHM) to simulate experimental resolution. 


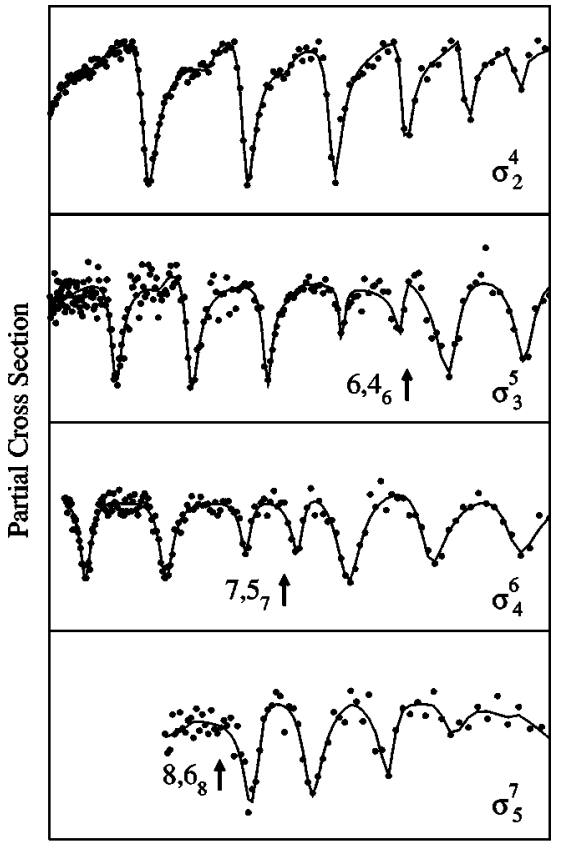

Scaled Photon Energy

FIG. 5. Partial photoionization cross sections $\sigma_{n}^{N}$ as a function of scaled photon energy. The vertical arrows mark the energies of the perturbers.

In general, an excellent agreement between experiment and theory is observed. However, in the theoretical PCSs $\sigma_{n}^{6}$ and $\sigma_{n}^{7}$, all spectral features were found at somewhat higher energies (by about $4 \mathrm{meV}$ and $12 \mathrm{meV}$ respectively), while for the PCSs $\sigma_{n}^{5}$ an almost perfect agreement was found.

\section{Propensity rules}

The propensity rules for autoionization of helium give predictions for the linewidths of doubly excited resonances and the general patterns of the PCSs. They are based on a molecular adiabatic description of $\mathrm{He}$ (analogous to $\mathrm{H}_{2}^{+}$) [26] and are derived from the changes in the nodal structure using the parabolic quantum numbers $N_{1}, N_{2}$, and $m$. In this way, three propensity rules for the autoionization process can be derived. The different propensity rules are caused by different decay mechanisms and are given in the order of the efficiency of the underlying decay mechanism:
(a) reduction of $N_{2}$,
(b) change of $m$,
(c) reduction of $N_{1}$.

The differences in the efficiency of the decay mechanisms are so strong that a decay via rule (b) or (c) shows only visible influence on the spectra if the decay processes on the basis of the preceding propensity rules are not possible. For a more detailed discussion of the propensity rules see Refs. $[5,11]$.

The coupling matrix elements, which lead to the propensity rules, are essentially very similar to the matrix elements that are relevant for the resonance parameters [27]. We can therefore conclude that resonances, which decay via the same propensity rule and have the same change in nodal structure, show similar line shapes. In particular, most of the spectral features in doubly excited helium are governed by propensity rule (a). In this case we expect similar line shapes for different resonances if the quantum number $N_{2}$ is changed by the same value, i.e., the changes of the nodal structure in radial wave functions are identical.

The total and the partial cross sections of doubly excited helium are dominated by the most intense principal series $N, K_{n}$, with $K=N-2$. These series can decay to all final states $\mathrm{He}^{+}(n)$ via the propensity rule (a) with the exception of $n$ $=1$. As a consequence, the general pattern should be visible in the PCSs $\sigma_{n}$ with $n \geqslant 2$. Applied to the quantum numbers $N, K_{n}$, the propensity rules for the PCSs predict that the decay of resonances $N, K_{n^{\prime}}$ to continuum states $n, k_{n^{\prime}}$ will result in similar patterns if $\Delta N_{2}=N_{2}-N_{2}^{\prime}=\Delta N=N-n=\Delta K=K-k$ $=1,2, \ldots$ has the same value. $N_{2}\left(N_{2}^{\prime}\right)$ represents the radial parabolic quantum number for the doubly excited (continuum) state and $k$ represents the angular correlation quantum number for the continuum state. This results in the expectation that the principal series below the $N=6$ ionization threshold exhibits the same structures in the $n=4 \mathrm{PCS}, \sigma_{4}^{6}$, as the principal series below the $N=5$ ionization threshold in the $n=3$ PCS, $\sigma_{3}^{5}$. This expectation was first confirmed by Menzel et al. [10], and it was called "general pattern."

Figure 5 presents examples of such cross section patterns, namely $\sigma_{2}^{4}, \sigma_{3}^{5}, \sigma_{4}^{6}$, and $\sigma_{5}^{7}$; in these PCSs, the resonances of the principal series decay by $\Delta N_{2}=N-n=2$. In order to demonstrate the general patterns, the photon energies were scaled to the effective quantum numbers $\mu_{N}$ by the Rydberg formula

$$
\mu_{N}(E)=\sqrt{\frac{\mathcal{R}}{I_{N}-E}},
$$

where $\mathcal{R}$ is the Rydberg constant. The similarities of the cross-section curves are evident except for the energy regions of perturber states indicated by vertical arrows. As mentioned above the perturber states affect the resonance energies and linewidths of the various resonances and, as a consequence, the regularities fade out in the vicinity of perturber states, but further away, the general pattern is restored. In the present measurements below the ionization threshold $I_{7}$, similar general patterns were observed in $\sigma_{4}^{5}$ and $\sigma_{5}^{6}$ with $\Delta N_{2}=1$, in $\sigma_{2}^{5}, \sigma_{3}^{6}$, and $\sigma_{4}^{7}$ with $\Delta N_{2}=3$, as well as in $\sigma_{2}^{6}$ and $\sigma_{3}^{7}$ with $\Delta N_{2}=4$. However, in the energy region of the higher ionization thresholds, such as in the $I_{7}$ manifold, the general patterns become very weak due to the complex structures caused by interferences with two additional perturber states that interact with the principal Rydberg series.

\section{Mirroring behavior and mirroring compensation properties}

According to Ref. [25], a PCS denoted by $P$ can be described analytically by

$$
\begin{aligned}
\sigma_{P}= & \sigma_{P}^{0}+\frac{\sigma_{P}^{0}}{1+\epsilon^{2}}\left\{2 \epsilon\left(q \operatorname{Re}\langle\alpha\rangle_{P}-\operatorname{Im}\langle\alpha\rangle_{P}\right)-2 q \operatorname{Im}\langle\alpha\rangle_{P}\right. \\
& \left.-2 \operatorname{Re}\langle\alpha\rangle_{P}+\left(q^{2}+1\right)\left\langle|\alpha|^{2}\right\rangle_{P}\right\} .
\end{aligned}
$$

Here, $\sigma_{P}^{0}$ is the PCS in the absence of a resonance and the 
second term describes the Fano-like line shape of the resonance in $\sigma_{P} .\langle\alpha\rangle_{P}$ and $\left\langle|\alpha|^{2}\right\rangle_{P}$ are the Starace parameters [25].

If the TCS is regarded as the sum of two random groups of PCSs, denoted by $P$ and $Q$, the PCS $P$ can be expressed by Eq. (7), and for the PCS $Q$ the same formula with $P$ replaced by $Q$ can be used. Note that PCS $P$ and PCS $Q$ can be individual PCSs or the sum of a number of individual PCSs, which in the present case are the $\sigma_{n}$. By using the formulas that establish relations between the parameters $\rho$ and $\sigma_{T}^{0}$ in the Fano formula for the TCS and the parameters $\sigma_{P}^{0}, \sigma_{Q}^{0}$, and $\alpha$ in the Starace formulas for the PCSs, namely, Eqs. (36), (40), (41), and (42) in Ref. [25], the PCS $Q$ can be written as (see the Appendix for its derivation)

$$
\begin{aligned}
\sigma_{Q}= & \sigma_{Q}^{0}-\frac{\sigma_{P}^{0}}{1+\epsilon^{2}}\left\{2 \epsilon\left(q \operatorname{Re}\langle\alpha\rangle_{P}-\operatorname{Im}\langle\alpha\rangle_{P}\right)-2 q \operatorname{Im}\langle\alpha\rangle_{P}\right. \\
& \left.-2 \operatorname{Re}\langle\alpha\rangle_{P}+\left(q^{2}+1\right)\left\langle|\alpha|^{2}\right\rangle_{P}\right\} \\
& +\frac{2 q \rho^{2} \sigma_{T}^{0}}{1+\epsilon^{2}} \epsilon+\frac{q^{2} \rho^{2} \sigma_{T}^{0}}{1+\epsilon^{2}}-\frac{\rho^{2} \sigma_{T}^{0}}{1+\epsilon^{2}} .
\end{aligned}
$$

The second term mirrors the line shape of the resonance in $\sigma_{P}$ and the last three terms describe the Fano-like line shape of the resonance in the total cross section. The contributions of the last three terms in Eq. (8) determine whether the resonances in the PCS $P$ and PCS $Q$ exhibit a mirroring behavior. The resonances in the two groups $P$ and $Q$ of the PCSs mirror each other if the second term of Eq. (8) is dominant as compared to the last three terms. If $|q| \gg 1$ and $\rho^{2}$ $\rightarrow 0$, the third and fifth terms in Eq. (8) can be neglected in comparison with the fourth term. In this case, Eq. (8) results in Eq. (8) in Ref. [13] if one omits, in addition to the third and fifth terms, all contributions of the second term that do not include $q$ or $q^{2}$. As discussed in Ref. [13], in this case the resonances display a Lorentzian profile in the TCS. In addition, a mirroring behavior for the resonances in $\sigma_{P}$ and $\sigma_{Q}$ has been predicted in Ref. [13].

In the region between the thresholds $I_{5}$ and $I_{7}$ of $\mathrm{He}^{+}$, the Fano parameter $\rho^{2}$ is of the order of 0.01 for the principal series [see Fig. 6(a) for the theoretical TCS below $I_{6}$ ]. In the other tablets of Figs. 6, we plot two examples, with the TCS $\sigma_{T}=\sigma_{P}+\sigma_{Q}$ being separated into two groups. In the first example $\sigma_{P}=\sigma_{1}$ and $\sigma_{Q}=\sigma_{2}+\sigma_{3}+\sigma_{4}+\sigma_{5}(\mathrm{~b} 1, \mathrm{~b} 2)$; in the second example, $\sigma_{P}=\sigma_{2}$ and $\sigma_{Q}=\sigma_{1}+\sigma_{3}+\sigma_{4}+\sigma_{5}$ (c1,c2). In the second example, the PCS $\sigma_{P}$ mirrors the PCS $\sigma_{Q}$ very well [Figs. 6(c1) and 6(c2)]. In contrast to this, the mirroring behavior is not well expressed in the first example [Figs. 6(b1) and 6(b2)]. This can be understood as follows: A mirroring behavior is expected if the amplitudes of the resonances in the TCS are small compared to variations in the PCS, i.e., if terms three to five in Eq. (8) are small compared to term two. In helium, however, each single PCS $\sigma_{n}$ exhibits amplitudes that are of the same order of magnitude as in $\sigma_{T}$. Since this holds for all ionization thresholds $I_{N}$ up to $N=9$ (see Fig. 3 in Ref. [11]), no general mirroring behavior will be expected in the double-excitation region of helium. Mirroring behavior occurs only accidentally, e.g., if the PCS $\sigma_{P}$ mirrors $\sigma_{T}$. In this case, the second term in Eq. (8) mimics $\sigma_{T}$ and a mir-

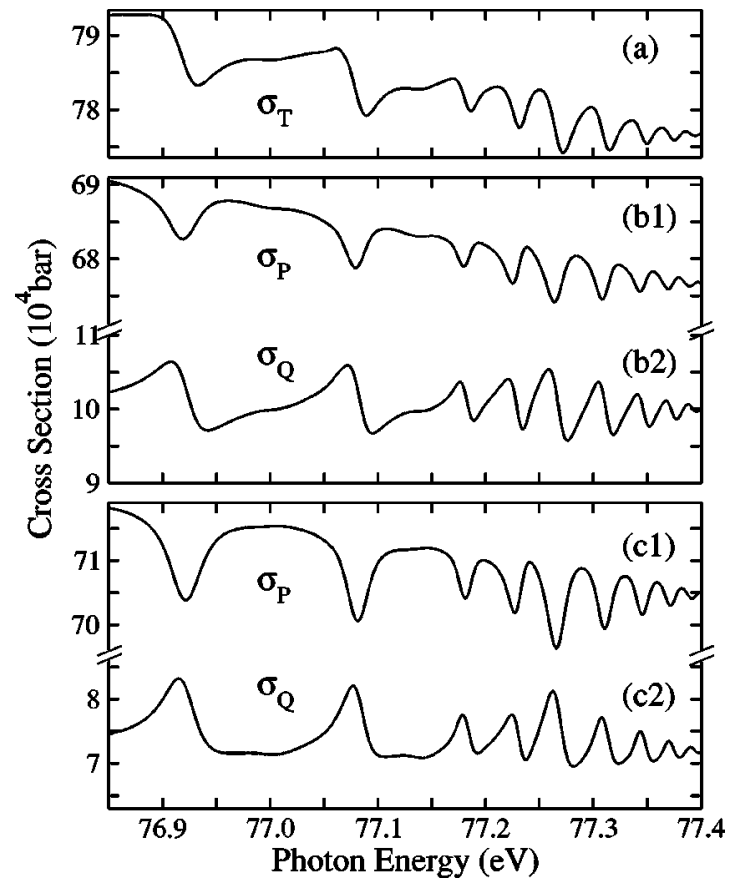

FIG. 6. Cross sections below the ionization threshold $I_{6}$ obtained from eigenchannel $R$ matrix calculations and convoluted with a Gaussian resolution function of $6 \mathrm{meV}$ width (FWHM). (a) Total cross section $\sigma_{T}$. In (b) and (c) the PCS $\sigma_{P}$ and $\sigma_{Q}$ were established in different ways: (b1) $\sigma_{P}=\sigma_{1}$ and (b2) $\sigma_{Q}=\sigma_{2}+\sigma_{3}$ $+\sigma_{4}+\sigma_{5} ;$; 1 ) $\sigma_{P}=\sigma_{1}+\sigma_{3}+\sigma_{4}+\sigma_{5}$ and (c2) $\sigma_{Q}=\sigma_{2}$.

roring behavior is observed. Even in cases where $\sigma_{P}$ is established by a number of $\sigma_{n}$, no general mirroring behavior is expected since the amplitude of the variation in $\sigma_{P}$ will still not be large as compared to the variation in $\sigma_{T}$. In summary, for the existence of a general mirroring behavior the condition $\rho^{2} \rightarrow 0$ is not sufficient. In addition to this it is also necessary that the amplitudes of resonances in the PCS are considerably larger than those in the TCS. However, as observed in the theoretical cross sections up to ionization threshold $I_{9}$ [11], the amplitudes in the PCS decrease by the same amount with increasing $N$ as the amplitudes in the TCS. As a consequence, we do not expect a general mirroring behavior in helium below higher- $N$ ionization thresholds $I_{N}$ with $\rho^{2}$ even much smaller than 0.01 .

As a next step, we define the "fractional partial cross sections" $\gamma_{P}:=\sigma_{P} / \sigma_{T}$ and $\gamma_{Q}:=\sigma_{Q} / \sigma_{T}$, i.e., we divide Eqs. (7) and (8) by $\sigma_{T}$. With $\gamma_{P}+\gamma_{Q}=\left(\sigma_{P}+\sigma_{Q}\right) / \sigma_{T}=1$, we obtain $\gamma_{Q}=1-\sigma_{P} / \sigma_{T}$ and find an exact mirroring behavior in the fractional partial cross sections. Figure 7 presents the same two examples as shown in Fig. 6, however, with $\gamma_{P}$ and $\gamma_{Q}$ instead of $\sigma_{P}$ and $\sigma_{Q}$. We see that in both cases the fractional partial cross sections $\gamma_{P}$ and $\gamma_{Q}$ exhibit perfect mirroring behavior, which can be considered as a mirroring compensation property between any two groups.

Since the sum of any two PCSs can be expressed mathematically by the Fano formula, the analysis given above can also be employed to discuss and understand the mirroring behavior of any two individual PCSs although the sum of these will not be the TCS, such as in case of $\sigma_{2}^{6}$ and $\sigma_{4}^{6}$ as well as $\sigma_{3}^{6}$ and $\sigma_{5}^{6}$ (see Fig. 3). In these cases, the resonances 


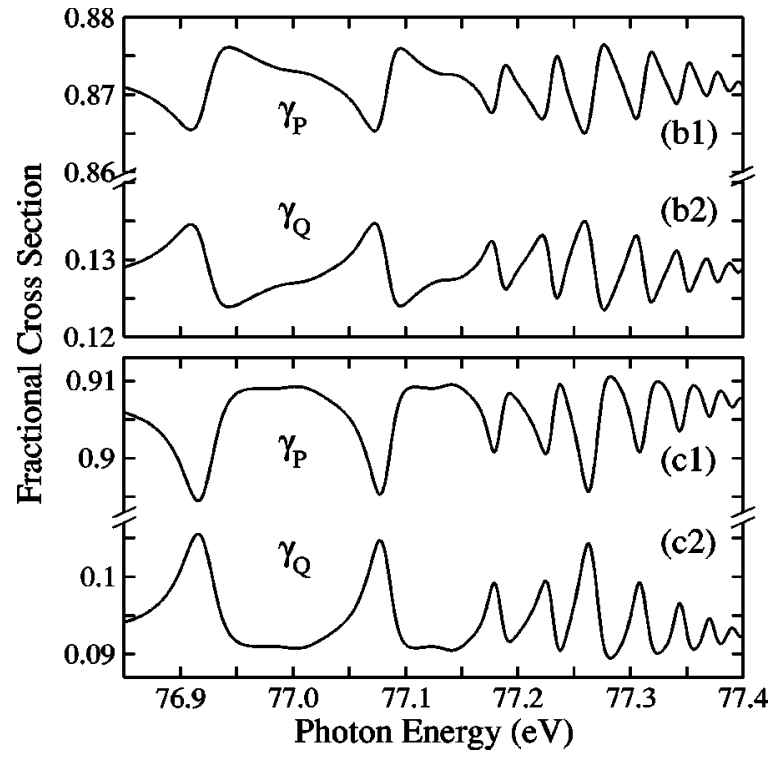

FIG. 7. Fractional partial cross sections $\gamma_{P}=\sigma_{P} / \sigma_{T}$ and $\gamma_{Q}$ $=\sigma_{Q} / \sigma_{T}$ below the ionization threshold $I_{6}$ obtained from eigenchannel $R$-matrix calculations and convoluted with a Gaussian resolution of $6 \mathrm{meV}$ width (FWHM).

in $\sigma_{S}=\sigma_{2}^{6}+\sigma_{4}^{6}$ and $\sigma_{S}=\sigma_{3}^{6}+\sigma_{5}^{6}$ are almost canceled out, and the $\sigma_{S}$ are expected to be quite structureless. On the other hand, if any $\sigma_{S}$ shows considerable structure, the contributing individual partial cross sections do not show mirroring behavior. From the above discussion we can also conclude that any two PCSs must display exact mirroring behavior if they are divided by their sum. Note that no limits for the parameters $\rho^{2}$ and $q$ are needed for this mirroring compensation property. In addition, the perturbers have no effect on the mirroring behavior between the PCS $P$ and PCS $Q$ and the mirroring compensation property.

In addition, it should be noted that below the ionization thresholds $I_{5}$ to $I_{7}$, the PCS $\sigma_{2}^{N}$ mirrors the PCS $\sigma_{4}^{N}$ and the PCS $\sigma_{3}^{N}$ mirrors the PCS $\sigma_{5}^{N}$ (not for $N=5$ ). This behavior might be due to a relation between the propensity rules and the mirroring behavior and can be an interesting topic for future studies.

\section{CONCLUSIONS}

In the present work, the partial cross sections $\sigma_{n}$ were measured in the energy region up to the ionization threshold $I_{7}$ and calculated by eigenchannel $R$-matrix theory. In general, the experimental data agree well the theoretical results and confirm the general patterns in the PCSs $\sigma_{n}$ derived from the propensity rules. The theoretical prediction that any two groups of PCSs - with the sum of those groups being the TCS - exhibit mirroring behavior if $\rho^{2} \rightarrow 0$ [13] is only partially observed. This can be understood by a more general consideration of the formulas given by Liu and Starace [13] and the fact that the variations in the PCSs and the TCS caused by the resonances are of the same order of magnitude. Instead, a mirroring compensation behavior of the abovementioned two groups is introduced and discussed.

\section{ACKNOWLEDGMENTS}

This work was supported by the Bundesministerium für Bildung und Forschung, project No. $05 \mathrm{KS} 1 \mathrm{~EB} 1 / 2$, and the Deutsche Forschnungsgemeinschaft, project No. PU 180/1-1. The authors appreciate the experimental support of Dr. M. Martins in the early stages of the work. The input by C.-N. Liu and T. Schneider at the early stages of this work is also gratefully acknowledged. Y.H.J. thanks the MPI für Physik Komplexer Systeme, Dresden, for financial support. U.B. gratefully acknowledges fruitful discussions with Dr. N. M. Kabachnik and Dr. A. Starace.

APPENDIX: DERIVATION OF EQUATION (8)

With Eqs. (36), (41), and (42) of Ref. [25], namely,

$$
\begin{gathered}
\sigma_{P}^{0}\left\langle|\alpha|^{2}\right\rangle_{P}+\sigma_{Q}^{0}\left\langle|\alpha|^{2}\right\rangle_{Q}=\rho^{2} \sigma_{T}^{0}, \\
\sigma_{P}^{0} \operatorname{Re}\langle\alpha\rangle_{P}+\sigma_{Q}^{0} \operatorname{Re}\langle\alpha\rangle_{Q}=\rho^{2} \sigma_{T}^{0},
\end{gathered}
$$

and

$$
\sigma_{P}^{0} \operatorname{Im}\langle\alpha\rangle_{P}-\sigma_{Q}^{0} \operatorname{Re}\langle\alpha\rangle_{Q}=0,
$$

Eq. (8) can be derived from Eq. (7) as follows:

$$
\begin{aligned}
\sigma_{Q}= & \frac{\sigma_{Q}^{0}}{1+\epsilon^{2}}\left\{\epsilon^{2}+2 \epsilon\left(q \operatorname{Re}\langle\alpha\rangle_{Q}-\operatorname{Im}\langle\alpha\rangle_{Q}\right)+\left[1-2 q \operatorname{Im}\langle\alpha\rangle_{Q}-2 \operatorname{Re}\langle\alpha\rangle_{Q}+\left(q^{2}+1\right)\left\langle|\alpha|^{2}\right\rangle Q\right]\right\} \\
= & \sigma_{Q}^{0}+\frac{\sigma_{Q}^{0}}{1+\epsilon^{2}}\left\{2 \epsilon\left(q \operatorname{Re}\langle\alpha\rangle_{Q}-\operatorname{Im}\langle\alpha\rangle_{Q}\right)+\left[-2 q \operatorname{Im}\langle\alpha\rangle_{Q}-2 \operatorname{Re}\langle\alpha\rangle_{Q}+\left(q^{2}+1\right)\left\langle|\alpha|^{2}\right\rangle_{Q}\right]\right\} \\
= & \sigma_{Q}^{0}+\frac{\sigma_{P}^{0}}{1+\epsilon^{2}}\left\{2 \epsilon\left(-q \operatorname{Re}\langle\alpha\rangle_{P}+\operatorname{Im}\langle\alpha\rangle_{P}+q \rho^{2} \sigma_{T}^{0} / \sigma_{P}^{0}\right)+\left[2 q \operatorname{Im}\langle\alpha\rangle_{P}+2 \operatorname{Re}\langle\alpha\rangle_{P}-2 \rho^{2} \sigma_{T}^{0} / \sigma_{P}^{0}-\left(q^{2}+1\right)\left\langle|\alpha|^{2}\right\rangle_{P}\right]\right. \\
& \left.+\left(q^{2}+1\right) \rho^{2} \sigma_{T}^{0} / \sigma_{P}^{0}\right\} \\
= & \sigma_{Q}^{0}-\frac{\sigma_{P}^{0}}{1+\epsilon^{2}}\left\{2 \epsilon\left(q \operatorname{Re}\langle\alpha\rangle_{P}-\operatorname{Im}\langle\alpha\rangle_{P}\right)-2 q \operatorname{Im}\langle\alpha\rangle_{P}-2 \operatorname{Re}\langle\alpha\rangle_{P}+\left(q^{2}+1\right)\left\langle|\alpha|^{2}\right\rangle_{P}\right\}+\frac{2 q \rho^{2} \sigma_{T}^{0}}{1+\epsilon^{2}} \epsilon+\frac{q^{2} \rho^{2} \sigma_{T}^{0}}{1+\epsilon^{2}}-\frac{\rho^{2} \sigma_{T}^{0}}{1+\epsilon^{2}} .
\end{aligned}
$$


[1] R. P. Madden and K. Codling, Phys. Rev. Lett. 10, 516 (1963).

[2] M. Domke, K. Schulz, G. Remmers, G. Kaindl, and D. Wintgen, Phys. Rev. A 53, 1424 (1996).

[3] R. Püttner, B. Grémaud, D. Delande, M. Domke, M. Martins, A. S. Schlachter, and G. Kaindl, Phys. Rev. Lett. 86, 3747 (2001).

[4] J.-Z. Tang and I. Shimamura, Phys. Rev. A 50, 1321 (1994).

[5] J. M. Rost, K. Schulz, M. Domke, and G. Kaindl, J. Phys. B 30, 4663 (1997).

[6] B. Grémaud, D. Delande, Europhys. Lett. 40, 363 (1997).

[7] H. Friedrich, Theoretical Atomic Physics (Springer, Berlin, 1998).

[8] D. W. Lindle, T. A. Ferrett, U. Becker, P. H. Kobrin, C. M. Truesdale, H. G. Kerkhoff, and D. A. Shirley, Phys. Rev. A 31, 714 (1985).

[9] A. Menzel, S. P. Frigo, S. B. Whitfield, C. D. Caldwell, M. O. Krause, J. Z. Tang, and I. Shimamura, Phys. Rev. Lett. 75, 1479 (1995).

[10] A. Menzel, S. P. Frigo, S. B. Whitfield, C. D. Caldwell, and M. O. Krause, Phys. Rev. A 54, 2080 (1996).

[11] T. Schneider, Ch. N. Liu, and J. M. Rost, Phys. Rev. A 65, 042715 (2002).

[12] H. W. van der Hart and C. H. Greene, Phys. Rev. A 66, 022710 (2002).

[13] Ch. N. Liu and A. F. Starace, Phys. Rev. A 59, R1731 (1999).

[14] U. Fano and J. W. Cooper, Rev. Mod. Phys. 40, 441 (1968), Sec. 8.1.
[15] S. E. Canton-Rogan, A. A. Wills, T. W. Gorczyca, M. Wiedenhoeft, O. Nayandin, Ch. N. Liu, and N. Berrah, Phys. Rev. Lett. 85, 3113 (2000).

[16] M. Martins, G. Kaindl, and N. Schwentner, J. Electron Spectrosc. Relat. Phenom. 101-103, 965 (1999).

[17] U. Becker, D. Szostak, H. G. Kerkhoff, M. Kupsch, B. Langer, R. Wehlitz, A. Yagishita, and T. Hayaishi, Phys. Rev. A 39, 3902 (1989).

[18] O. Hemmers, S. B. Whitfield, P. Glans, H. Wang, and D. W. Lindle, R. Wehlitz, and I. A. Sellin, Rev. Sci. Instrum. 69, 3809 (1998).

[19] P. F. O'Mahony and C. H. Greene, Phys. Rev. A 31, 250 (1985).

[20] C. Pan, A. F. Starace, and C. H. Greene, Phys. Rev. A 53, 840 (1996).

[21] A. F. Starace, in Corpuscles and Radiation in Matter, edited by W. Mehlhorn, Handbuch der Physik Vol. 31 (Springer, Berlin, 1982).

[22] D. R. Herrick and O. Sinanoğlu, Phys. Rev. A 11, 97 (1975).

[23] C. D. Lin, Phys. Rev. A 29, 1019 (1984).

[24] U. Fano and J. W. Cooper, Phys. Rev. 137, A1364 (1965).

[25] A. F. Starace, Phys. Rev. A 16, 231 (1977).

[26] J. M. Feagin and J. S. Briggs, Phys. Rev. Lett. 57, 984 (1986).

[27] T. Schneider, Doctoral Thesis, Max-Planck-Institut für Physik Komplexer Systeme, Dresden, Germany, 2002 (unpublished). 